\title{
Ceftolozane/Tazobactam-Induced Leukocytosis and Clinical Failure in a Patient Being Treated for Ventilator-Associated Pneumonia Caused by Carbapenem-Resistant Pseudomonas aeruginosa: a Case Report
}

\author{
Bejoy P. Maniara ${ }^{1}$ (D) $\cdot$ Ian Wells ${ }^{1}$
}

Accepted: 10 January 2021 / Published online: 21 January 2021

(C) The Author(s), under exclusive licence to Springer Nature Switzerland AG part of Springer Nature 2021

\begin{abstract}
Ceftolozane/tazobactam is an intravenous beta-lactam/beta-lactamase inhibitor that utilizes a novel oxyimino-cephalosporin with a traditional beta-lactamase inhibitor. It is approved by the Food and Drug Administration to treat complicated intra-abdominal infections in combination with metronidazole, complicated urinary tract infections, and, most recently, hospital-acquired bacterial and ventilator-associated bacterial pneumonias. It is commonly utilized to treat infections caused by multidrug-resistant Pseudomonas aeruginosa. This case report delineates the first published case of ceftolozane/tazobactam-induced leukocytosis (up to $36.9 \times 10^{9}$ cells $/ \mathrm{L}$ ) and clinical failure when utilized in a high-dose regimen for a patient being treated for ventilatorassociated pneumonia secondary to carbapenem-resistant $P$. aeruginos $a$. The reaction occurred during initial challenge, resolved after discontinuation, and recurred during re-challenge. In patients who are appropriately being treated with ceftolozane/ tazobactam for susceptible infections, consider a drug-induced reaction as a potential cause of rising leukocytosis; this should be differentiated from clinical failure if the patient is clinically stable.
\end{abstract}

Keywords Ceftolozane/tazobactam $\cdot$ Drug-induced $\cdot$ Idiosyncratic $\cdot$ Leukocytosis $\cdot$ Failure

\section{Introduction}

Pseudomonas aeruginosa is a highly pathogenic Gramnegative bacterium that may be difficult to treat due to various intrinsic and acquired mechanisms of resistance. The predominant intrinsic mechanisms of resistance include poor outer membrane permeability due to various restrictive porins (e.g., OprD), expressed efflux pumps (e.g., MexAB-OprM, MexCD-OprJ, MexEF-OprN, and MexXY-OprM), and antibiotic-deactivating enzymes (e.g., AmpC betalactamases). Although $P$. aeruginosa has potential to produce beta-lactamases from all four Ambler classes, it does not

This article is part of the Topical Collection on Medicine

Bejoy P. Maniara

bejoymaniara@gmail.com

Ian Wells

iwells@kingsbrook.org

1 Present address: Department of Pharmacy, Kingsbrook Jewish Medical Center, 585 Schenectady Ave, Brooklyn, NY 11203, USA commonly produce these enzymes with the exception of class C (e.g., AmpC) [1]. Resistance mechanisms may also be acquired through mobile genetic elements (e.g., plasmids, transposons, and integrons) that harbor resistance genes. Mobile genetic elements may be transferred from one bacterium to another via horizontal gene transfer (i.e., transformation, transduction, and conjugation). This may cause loss of porin channels, hyperproduction of efflux pumps, and/or production of antibiotic-deactivating enzymes in $P$. aeruginosa. These resistance mechanisms may prevent effective use of most anti-pseudomonal beta-lactams, including anti-pseudomonal carbapenems [2, 3]. Unfortunately, antimicrobials that can bypass all mechanisms of resistance do not currently exist. Ceftolozane/tazobactam, a novel oxyimino-aminothiazolyl cephalosporin, has the ability to bypass certain betalactamase (i.e., chromosomal AmpC), porin (i.e., OprD), efflux pump (i.e., MexXY-OprM, MexAB-OprM) mutations commonly identified in $P$. aeruginosa, due to its structural stability against these mechanisms, higher affinity towards $P$. aeruginosa penicillin binding proteins (i.e., $1 \mathrm{~b}, 1 \mathrm{c}, 2$, and 3 ), and the presence of tazobactam to inhibit certain Ambler class $\mathrm{A}$ and $\mathrm{C}$ beta-lactamases $[2,4-6]$. 
Ceftolozane/tazobactam is a novel intravenous beta-lactam/beta-lactamase inhibitor combined with a traditional beta-lactamase inhibitor that was approved by the US Food and Drug Administration (FDA) as monotherapy for complicated urinary tract infections and for complicated intraabdominal infections when used concomitantly with metronidazole. For both indications, ceftolozane/tazobactam is dosed as $1.5 \mathrm{~g}$ intravenously (IV) every $8 \mathrm{~h}$. It recently received FDA approval for hospital-acquired pneumonia (HAP) and ventilator-associated pneumonia (VAP), but it must be dosed at $3 \mathrm{~g}$ IV every $8 \mathrm{~h}$ [7]. Ceftolozane/tazobactam demonstrates higher concentrations in lung epithelial lining fluid (ELF) than piperacillin/tazobactam demonstrates, but doses of $1.5 \mathrm{~g}$ every $8 \mathrm{~h}$ did not produce adequate lung ELF concentrations to eradicate $P$. aeruginosa as plasma-to-ELF penetration ratio is approximately $50 \%$. Dosing $1.5 \mathrm{~g}$ IV every $8 \mathrm{~h}$ produced a mean ceftolozane ELF concentration of $21.8 \mathrm{mg} / \mathrm{L}$, which may not be sufficient in multidrug-resistant $P$. aeruginosa $[8$, 9]. Several studies and case reports demonstrate that doubling the dose to $3 \mathrm{~g}$ IV every $8 \mathrm{~h}$ may be sufficient to obtain adequate lung ELF concentrations over the dosing interval to achieve $>90 \%$ probability of target attainment and treat nosocomial pneumonia secondary to multidrug-resistant P. aeruginosa [9-15].

Ceftolozane/tazobactam failures have been observed when used off-label in pneumonia. Many of these patients are more severely ill compared to patients who experienced clinical success [15]. Currently, there are not any reported cases of ceftolozane/tazobactam-induced leukocytosis. Herein, we report the first published case of ceftolozane/tazobactaminduced leukocytosis and clinical failure when utilized for a patient being treated for VAP secondary to carbapenemresistant $P$. aeruginosa, and during re-challenge.

\section{Case Presentation}

We present the case of a 25-year-old Caucasian male with quadriplegia who was bedridden in a nursing home. He presented with fever and tachycardia and was diagnosed with sepsis. Blood and tracheostomy secretion samples were sent for culture and susceptibility testing in the nursing home. Symptom assessment was difficult due to medical history of a gunshot wound leading to quadriplegia and anoxic brain injury. A chest X-ray demonstrated right upper lobe pneumonia. He was empirically initiated on vancomycin $750 \mathrm{mg}$ IV every $8 \mathrm{~h}$, cefepime $1000 \mathrm{mg}$ IV every $8 \mathrm{~h}$, and metronidazole $500 \mathrm{mg}$ IV every $8 \mathrm{~h}$ on day 1 , and subsequently transferred to the intensive care unit (ICU). Fever (temperature $101.1^{\circ} \mathrm{F}$ ), tachycardia (heart rate 137 beats per minute (bpm)), tachypnea (respiratory rate 29 breaths per minute (bpm)), and leukocytosis (white blood cell (WBC) count $21 \times 10^{9} / \mathrm{L}$ ) were documented on ICU presentation.
Respiratory secretions grew pan-susceptible Pseudomonas aeruginosa and blood cultures grew methicillin-resistant Staphylococcus epidermidis; empiric antibiotics were continued with positive clinical response. Fever defervesced, blood cultures became negative on day three, and leukocytosis, tachycardia, and tachypnea were gradually resolving. A repeat chest X-ray demonstrated large right-sided hydropneumothorax and increased diffuse left-sided airspace opacities. A right-sided chest tube was subsequently placed.

On day 10, vancomycin was discontinued, and linezolid was initiated for 12 additional days due to persistent signs and symptoms. A non-contrast chest computed tomography (CT) later demonstrated probable mucus plug and possible postobstructive pneumonia. On day 22, our patient was downgraded. A repeat respiratory culture then grew heavy Acinetobacter baumannii-calcoaceticus and moderate $P$. aeruginosa with many WBCs, so linezolid was discontinued. On day 24, the chest tube was removed and there was not any evidence of pneumothorax following removal.

On day 25 , both $A$. baumannii and $P$. aeruginosa were found to be resistant to cefepime and imipenem; cefepime was discontinued. Additional susceptibilities and molecular testing for carbapenemases were requested for both organisms. Meanwhile, our patient was initiated on dualcarbapenem therapy (ertapenem $1000 \mathrm{mg}$ IV every $24 \mathrm{~h}$ and meropenem $500 \mathrm{mg}$ IV every $6 \mathrm{~h}$ ) to combat potential OXA48 carbapenemases in A. baumannii, polymyxin B 800,000 units IV every $12 \mathrm{~h}$, and colistin $150 \mathrm{mg}$ inhaled every $8 \mathrm{~h}$ to synergistically combat potential porin and efflux pump mutations. Acinetobacter baumannii was found to be susceptible to tetracycline, so minocycline $200 \mathrm{mg}$ orally every $12 \mathrm{~h}$ was initiated. On day 32, blood cultures grew yeast, so micafungin $150 \mathrm{mg}$ IV every $24 \mathrm{~h}$ was initiated. The culture was later confirmed as Candida auris. Beyond day 32, surveillance blood cultures were negative.

While on ertapenem, meropenem, polymyxin B, inhaled colistin, minocycline, and micafungin, our patient seemed to be improving. However, on day 35, our patient went into cardiac arrest. On day 38, our patient's WBC decreased to $14.3 \times 10^{9} / \mathrm{L}$, the lowest since initiating this regimen. A respiratory culture demonstrated light growth of $P$. aeruginosa with moderate WBCs.

On day 38 , both $A$. baumannii and $P$. aeruginosa were found to be susceptible to polymyxin B and colistin; $P$. aeruginosa was also susceptible to ceftolozane/tazobactam. Molecular testing was unable to be performed. Ertapenem, meropenem, polymyxin $\mathrm{B}$, inhaled colistin, and oral minocycline were discontinued, and ceftolozane/tazobactam $3 \mathrm{~g}$ IV every $8 \mathrm{~h}$, for carbapenem-resistant $P$. aeruginos $a$, and minocycline $100 \mathrm{mg}$ IV every $12 \mathrm{~h}$, for carbapenem-resistant A. baumannii, were initiated, and micafungin was continued. Our patient's WBCs progressively increased from $14.3 \times 10^{9} /$ $\mathrm{L}$ on day 38 to $26.2 \times 10^{9} / \mathrm{L}$ on day 42 . Our patient also 
Fig. 1 The trend in white blood cell count throughout duration of antimicrobial therapy, including when ceftolozane/tazobactam was started and stopped, demonstrating drug-induced leukocytosis. Figure created in Microsoft Excel

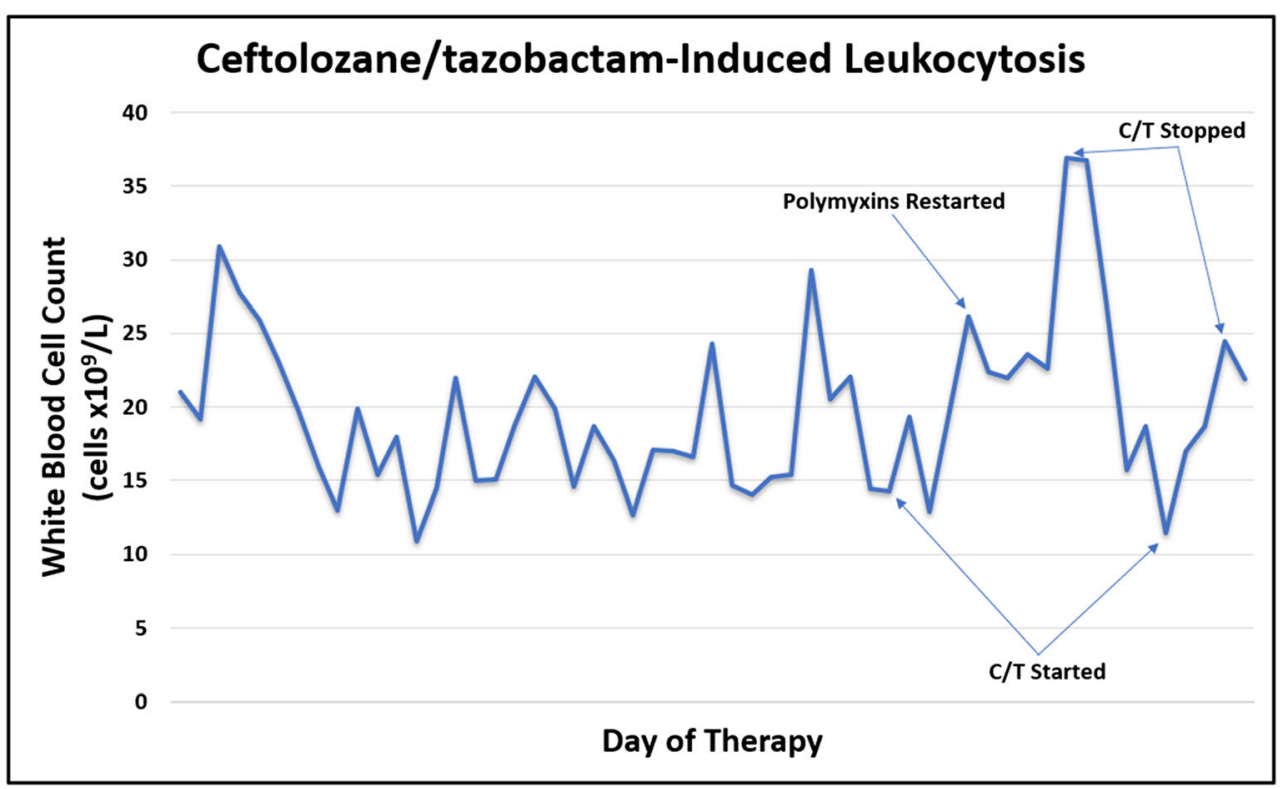

became tachycardic to $129 \mathrm{bpm}$, tachypneic to $32 \mathrm{bpm}$, and febrile to $100.8^{\circ} \mathrm{F}$, so intravenous polymyxin $\mathrm{B}$ and inhaled colistin were re-initiated A repeat respiratory culture persistently grew $P$. aeruginosa. On day 48 , our patient's WBCs peaked at $36.9 \times 10^{9} / \mathrm{L}$.

On day 50, all antimicrobials were discontinued, and WBC declined to $26.9 \times 10^{9} / \mathrm{L}$. A non-contrast chest $\mathrm{CT}$ demonstrated worsening left lower lobe pneumonia without abscess and severe bronchiectasis with mucus plugging, but our patient's WBC further improved to $11.5 \times 10^{9} / \mathrm{L}$ on day 53 while off antimicrobials. Ceftolozane/tazobactam monotherapy was reinitiated due to radiographic findings.

Immediately after re-challenging ceftolozane/tazobactam, our patient's WBCs increased from $11.5 \times 10^{9}$ to $17 \times 10^{9} / \mathrm{L}$ on day $54,18.7 \times 10^{9} / \mathrm{L}$ on day 55 , and $24.5 \times 10^{9} / \mathrm{L}$ on day 56 (Fig. 1). A repeat respiratory culture again grew $P$. aeruginosa. Ceftolozane/tazobactam was discontinued and our patient's WBC improved to $21.9 \times 10^{9} / \mathrm{L}$. Our patient was then transferred to an outside facility. Unfortunately, our patient's status could not be tracked beyond the transfer.

\section{Conclusions}

To the best of our knowledge, this is the first reported case demonstrating ceftolozane/tazobactam-induced leukocytosis. Our patient initially appeared responsive to polymyxin-based therapy. When transitioned to ceftolozane/tazobactam, his WBCs began to worsen, though he remained clinically stable. We initially attributed this to clinical failure of ceftolozane/ tazobactam and added polymyxin B and colistin. Despite prolonged combination therapy with active antimicrobials, Pseudomonas aeruginosa microbiologic clearance was not achieved. Unfortunately, our patient's WBC continued to worsen. Upon discontinuation of all antimicrobials and monitoring for 3 days, our patient's WBCs improved. Upon reinitiation of ceftolozane/tazobactam monotherapy, our patient's WBCs rose again. Three days later, ceftolozane/ tazobactam was again discontinued and, once again, WBCs declined. According to the Naranjo algorithm, our patient experienced a probable drug-induced adverse drug reaction. Though it is unclear how ceftolozane/tazobactam may induce leukocytosis, clinical failure should be differentiated from an adverse drug reaction in patients with increasing leukocytosis but stable symptoms on ceftolozane/tazobactam.

Acknowledgements The collaborative contributions from the following individuals have been greatly appreciated: (1) Bejoy Maniara, PharmD, BCPS and (2) Ian Wells, BS, PharmD.

Author Contributions I (Dr. Bejoy Maniara) substantially contributed to the conception of this case report and acquisition, analysis, and interpretation of data. I drafted the article and critically revised it for important intellectual content. I have provided final approval of this version to be published. I agree to be accountable for all aspects of the work in ensuring that questions related to the accuracy or integrity of any part of the work are appropriately investigated and resolved.

Dr. Ian Wells also substantially contributed to the conception of this case report and acquisition, analysis, and interpretation of data. He assisted in drafting the article, as a co-author, and assisted in critically revising it for important intellectual content. He has provided final approval of this version to be published. He agrees to be accountable for all aspects of the work in ensuring that questions related to the accuracy or integrity of any part of the work are appropriately investigated and resolved.

\section{Compliance with Ethical Standards}

Conflict of Interest The authors declare that they have no conflict of interest. 
Ethics Approval The Institutional Review Board waived the need for ethical approval for this case report given that identifiable health information was not disclosed.

Informed Consent Consent to participate and publish this case report was obtained from the patient.

\section{References}

1. Yordanov D, Strateva T. Pseudomonas aeruginosa - a phenomenon of bacterial resistance. J Med Microbiol. 2009;58:1133-48.

2. Castanheira M, Mills JC, Farrell DJ, Jones RN. Mutation-driven $\beta$ lactam resistance mechanisms among contemporary ceftazidimenonsusceptible Pseudomonas aeruginosa isolates from U.S. hospitals. Antimicrob Agents Chemother. 2014;58:6844-50.

3. Pang Z, Raudonis R, Glick BR, Lin T-J, Cheng Z. Antibiotic resistance in Pseudomonas aeruginosa: mechanisms and alternative therapeutic strategies. Biotechnol Adv. 2019;37:177-92.

4. Hong M-C, Hsu DI, Bounthavong M. Ceftolozane/tazobactam: a novel antipseudomonal cephalosporin and $\beta$-lactamase-inhibitor combination. Infect Drug Resist. 2013;6:215-23.

5. Wi YM, Greenwood-Quaintance KE, Schuetz AN, Ko KS, Peck KR, Song J-H, et al. Activity of ceftolozane-tazobactam against carbapenem-resistant, non-carbapenemase-producing Pseudomonas aeruginosa and associated resistance mechanisms. Antimicrobial Agents Chemother [Internet]. 2018 [cited 2020 Jan 20];62. Available from: https://aac.asm.org/content/62/1/ e01970-17.

6. Zhanel GG, Chung P, Adam H, Zelenitsky S, Denisuik A, Schweizer F, et al. Ceftolozane/tazobactam: a novel cephalosporin/ $\beta$-lactamase inhibitor combination with activity against multidrug-resistant Gram-negative bacilli. Drugs. 2014;74:31-51.

7. zerbaxa pi [Internet]. [cited 2019 Aug 25]. Available from: https:// www.merck.com/product/usa/pi_circulars/z/zerbaxa/zerbaxa_pi. pdf.
8. Chandorkar G, Huntington JA, Gotfried MH, Rodvold KA, Umeh O. Intrapulmonary penetration of ceftolozane/tazobactam and piperacillin/tazobactam in healthy adult subjects. J Antimicrob Chemother. 2012;67:2463-9.

9. Xiao AJ, Miller BW, Huntington JA, Nicolau DP. Ceftolozane/ tazobactam pharmacokinetic/pharmacodynamic-derived dose justification for phase 3 studies in patients with nosocomial pneumonia. J Clin Pharmacol. 2016;56:56-66.

10. Castón JJ, De la Torre Á, Ruiz-Camps I, Sorlí ML, Torres V, TorreCisneros J. Salvage Therapy with ceftolozane-tazobactam for multidrug-Resistant Pseudomonas aeruginosa infections. Antimicrob Agents Chemother [Internet]. 2017 [cited 2019 Mar 2];61. Available from: https://www.ncbi.nlm.nih.gov/pmc/articles/ PMC5328537/.

11. Munita JM, Aitken SL, Miller WR, Perez F, Rosa R, Shimose LA, et al. Multicenter evaluation of ceftolozane/tazobactam for serious infections caused by carbapenem-resistant Pseudomonas aeruginosa. Clin Infect Dis. 2017;65:158-61.

12. Haidar G, Philips NJ, Shields RK, Snyder D, Cheng S, Potoski BA, et al. Ceftolozane-tazobactam for the treatment of multidrugresistant Pseudomonas aeruginosa infections: clinical effectiveness and evolution of resistance. Clin Infect Dis. 2017;65:110-20.

13. Gelfand MS, Cleveland KO. Ceftolozane/tazobactam therapy of respiratory infections due to multidrug-resistant Pseudomonas aeruginosa. Clin Infect Dis. 2015;61:853-5.

14. Safety and Efficacy Study of Ceftolozane/Tazobactam to Treat Ventilated Nosocomial Pneumonia (MK-7625A-008) - Full Text View - ClinicalTrials.gov [Internet]. [cited 2019 Mar 2]. Available from: https://clinicaltrials.gov/ct2/show/NCT02070757.

15. Lewis PO, Cluck DB, Tharp JL, Krolikowski MA, Patel PD. Failure of ceftolozane-tazobactam salvage therapy in complicated pneumonia with lung abscess. Clin Case Rep. 2018;6:1308-12.

Publisher's Note Springer Nature remains neutral with regard to jurisdictional claims in published maps and institutional affiliations. 